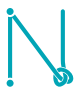

Volumen $5 \mathrm{~N} .{ }^{\circ} 42$ enero - junio de 2017 ISSN: 0122-4328

ISSN-E: 2619-6069 pp. 126-137
Docente de Educación Superior, Universidad Pedagógica Nacional, Unidad Ajusco. Miembro de la Red de Lenguaje México. Otra educación es posible. cruiznakasone@gmail.com

Docente de Educación Básica, Escuela Primaria en la Comunidad de Zoquiapam, Sierra Norte, Oaxaca.

Miembro de la Red de Lenguaje México. Otra educación es posible. vemafe.84@gmail.com

\section{La Red Lenguaje México. Otra educación es posible}

\author{
Formarnos para transformarnos: experiencias \\ en la formación de profesores desde la \\ Pedagogía por Proyectos
}

\author{
María del Carmen Ruíz Nakasone* \\ Lucerito Rosales Martínez**
}

\section{RESUMEN}

El trabajo "La red de Lenguaje México. Otra educación es posible. Formarnos para Transformarnos. Experiencias en la formación de profesores desde la Pedagogía por Proyectos" da cuenta de algunos procesos que vivimos en la formación de profesores de educación básica en el estado de Oaxaca, a partir de la propuesta Pedagogía por Proyectos. En este se da a conocer cómo desde la Red de Lenguaje asumimos la Pedagogía por Proyectos como una estrategia de formación para construir procesos alternativos para transformar nuestras aulas, escuelas y, sobre todo, nuestras prácticas de enseñanza.

A lo largo del texto compartimos algunos testimonios que dan cuenta sobre el proceso que los profesores vivieron desde otra perspectiva de formación, diferente a los cursos de actualización impartidos por instancias oficiales. Esta es una formación caracterizada por la construcción colectiva de proyectos de aula, el cambio en las relaciones verticales por relaciones horizontales, sus implicaciones en la manera de organizar el trabajo en el aula, en la toma de decisiones, en las maneras de enseñar y aprender, y en la manera de vivirnos como sujetos protagónicos del proceso educativo.

\section{Palabras clave}

Formación docente; Pedagogía por Proyectos; transformación; trabajo colectivo; relaciones horizontales 


\section{Presencia histórica de un accionar de Pilec y la Red de Lenguaje México. Otra educación es posible}

En 1991, un grupo de profesores de la Universidad Pedagógica Nacional, Unidad Ajusco, ${ }^{1}$ con una larga trayectoria de lucha político-sindical y académica nos propusimos impulsar un proyecto con docentes de educación básica del estado de Oaxaca y la Ciudad de México; nos referimos al Proyecto de Intervención Lectura y Escritura en Educación Básica (Pilec). La perspectiva de formación de maestros, desde el Pilec, ha considerado encuentros de experiencias entre docentes y otros hacedores de la cultura escrita, visitas de apoyo y acompañamiento a los profesores en sus lugares de trabajo, ferias del libro y sus lectores en comunidades marginadas, talleres y actividades de animación sociocultural con padres de familia y estudiantes. Estas acciones han fortalecido las prácticas en las escuelas y sus maestros en torno a la cultura escrita, así como en la reinvención de lo político-pedagógico entre docentes y sus comunidades.

Desde el proyecto Pilec sostenemos que los maestros son sujetos portadores de saberes pedagógicos y constructores de cultura, sujetos que generan propuestas pedagógicas innovadoras y abonan a la reflexión educativa. Desde esta concepción de profesores surge la necesidad de promover la construcción de proyectos colectivos de aula, escuela y comunidad, a través de la Pedagogía por Proyectos y la Documentación narrativa de las experiencias pedagógicas de los docentes, con el fin de relatar el acontecer de la escuela y las historias de maestros, contadas desde la voz de sus protagonistas.

Asumimos que los profesores, desde la documentación narrativa de sus experiencias pedagógicas, pueden aportar nuevas fuentes de conocimiento pedagógico, y con ello, enriquecer la realidad educativa desde la realidad de las aulas, desde la singularidad de sus escuelas y contextos, y desde la interacción de sus subjetividades. A partir de la narrativa ha sido posible promover la difusión de las experiencias pedagógicas innovadoras e historias de docentes que participan desde diversos contextos rurales y urbanos marginales.

Por otra parte, Pilec, desde 1996, ha sido un proyecto protagónico en la conformación de redes de maestros: la Red Transformación de la Educación Básica desde la Escuela (Red Tebes) constituida desde la UPN; la Red de Maestros Animadores de la Lectura, en Iztapalapa, CDMx; la Red Totoax en Totolapa, Tlacolula, Oaxaca (zona rural); la Red de Lenguaje Oaxaca; la Red Metropolitana de Lenguaje (CDMx y Estado de México); y la Red de Lectura y Escritura, Monclova, Coahuila (Red LEE, Monclova). Aquí descansa la identidad colectiva que, desde el Proyecto Pilec, se ha fomentado durante los 22 años de su quehacer, en la construcción colectiva de una propuesta de formación de profesores de educación básica.

1 Los profesores que participamos en la construcción del Proyecto PILEC fuimos Rigoberto González Nicolás, Roberto Pulido Ochoa y Carmen Ruiz Nakasone, académicos de la Universidad Pedagógica Nacional, Unidad Ajusco, CDMX. 


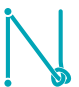

Volumen 5 N. ${ }^{\circ} 42$ enero - junio de 2017 ISSN: 0122-4328 ISSN-E: 2619-6069 pp. $126-137$

Actualmente, algunos maestros y maestras, que a lo largo de los años han participado en las distintas redes, nos hemos integrado en la Red de Lenguaje México. Otra educación es posible, conformada por seis nodos: Nodo Metropolitano-CDMx-Estado de México, Nodo Oaxaca-Oaxaca, Nodo Costa-Oaxaca, Nodo Tlaxiaco-Oaxaca, Nodo Monclova-Coahuila y Nodo Cherán-Michoacán.

Una de las propuestas de formación que actualmente impulsamos como Red de Lenguaje y desde la Universidad Pedagógica Nacional es la Pedagogía por Proyectos, cuyos propósitos son: la formación de los estudiantes y maestros en un ambiente democrático con sentido cooperativo y solidario, y la formación de lectores y productores de textos autónomos con sentido crítico.

Nuestro interés en este trabajo es dar a conocer algunos aspectos del proceso de formación que vivieron las y los profesores que participaron en dicho programa de formación. Iniciamos con un panorama general de la formación de profesores desde la política educativa, contexto desde el cual decidimos el desarrollo de la Pedagogía por Proyectos. Posteriormente, abordamos algunos elementos del proceso de transformación que vivieron las y los maestros participantes.

\section{La formación de profesores. Una manera de resistir}

En México vivimos, desde el 2013 (Aboites, 2012; Hernández, 2013), la imposición de una Reforma Educativa que no pretende mejorar la calidad en la educación, sino ejercer control sobre los maestros a través de reformas laborales y administrativas. Desde la Reforma Educativa se ha pretendido estandarizar, a lo largo del territorio mexicano, los programas curriculares con el fin de ajustar los fines educativos a los requerimientos establecidos por los organismos internacionales.

Desde hace años hemos sido testigos de cómo los cambios propuestos desde el sistema educativo mexicano no han considerado a los profesores como sujetos que participan en la elaboración de propuestas educativas:2 por el contrario, los han concebido como reproductores de programas curriculares definidos desde las políticas educativas.

Uno de los aspectos que hemos vivido en estos procesos de estandarización ha sido la formación continua que la Secretaría de Educación Pública (sep) ha llevado a cabo. Desde los cursos de capacitación, desde una estrategia en casacada, vertical y masiva, se ha pretendido ofrecer a los maestros modelos y herramientas pedagógicas para "mejorar sus estrategias de enseñanza", a partir de los planteamientos de la Reforma Educativa en turno. Desde esta lógica, los profesores cumplen con la enseñanza de los contenidos curriculares establecidos en los planes y programas oficiales sin considerar las experiencias pedagógicas de los maestros, las condiciones socioculturales de cada contexto y los saberes de las comunidades.

2 Durante el sexenio del 2006-2012 se establece un acuerdo denominado "La Alianza por la Calidad de la Educación" (ace), primer antecedente que hace alusión al ingreso y promoción del servicio docente. En el 2012, se firma el "pacto por México" que es un acuerdo político entre el gobierno federal y los partidos políticos del país que impulsa la puesta en marcha de tres reformas estructurales: comunicaciones, educativa y de hacienda. 
Como Red de Lenguaje asumimos que en los procesos de formación es necesaria la flexibilidad para hacer y construir desde el contexto en el que se trabaja y con las necesidades reales de niños y padres de familia. Consideramos los procesos de formación como un proceso de apropiación y transformación, con el que los maestros, como sujetos pensantes, generamos propuestas alternativas desde nuestras escuelas.

Consideramos que hay otras formas de hacer escuela, a partir el trabajo colectivo, como un acto de resistencia, con posibilidad de construir espacios y propuestas alternativas, desde abajo y desde adentro, desde la comunidad escolar, junto con los alumnos y padres de familia.

\section{La Red de Lenguaje, un proyecto para construir sentidos desde abajo, desde adentro, desde la Pedagogía por Proyectos}

Con la Red de Lenguaje y la Universidad Pedagógica Nacional ${ }^{3}$ hemos planteado programas de formación dirigidos a profesores de Educación Básica cuyo propósito ha sido la formación de niños y maestros como lectores y productores de textos. Uno de los programas que hemos desarrollado es la Pedagogía por Proyectos, cuya finalidad ha sido la formación permanente de profesores a partir de un programa que articula la teoría, la práctica docente y sus procesos didácticos (Ruíz et al., 2010).

Decidimos trabajar la Pedagogía por Proyectos porque es una estrategia de formación y una propuesta didáctica que promueve la construcción social de proyectos desde el aula y la comunidad, en un ambiente democrático y solidario, con la participación de maestros, alumnos y padres de familia. Con esta propuesta se pueden construir otras formas de ser maestros y de hacer escuela, a través de la autonomía y una perspectiva de trabajo horizontal, colaborativo, inclusivo e solidario, principios fundamentales de la Red de Lenguaje.

Construir "otras formas de ser maestros y hacer escuela" ha significado desaprender para aprender. Desaprender prácticas de enseñanza que han reproducido conocimientos como únicos, válidos y verdaderos (Palermo, 2014, p. 16), para aprender que hay diversidad de saberes circulando en el aula y la comunidad; desaprender a mirarnos como maestros pasivos, reproductores de currículum establecidos por la Secretaría de Educación Pública, para aprender a construir currículum con los saberes de los alumnos y por medio del contexto de la escuela y la comunidad; desaprender a vivir la relación unilateral con los padres de familia en la que solo nuestras decisiones definen el rumbo escolar de sus hijos, para aprender a relacionarnos como sujetos con historias e intenciones educativas comunes; desaprender a concebir a los alumnos como sujetos pasivos que solo reciben información y órdenes, para aprender a mirarlos como sujetos que poseen saberes, hacen propuestas y deciden el rumbo de sus aprendizajes. Nuestro propósito ha sido generar alternativas

3 La Red de Lenguaje México y la Universidad Pedagógica Nacional, Unidad Ajusco, a través del Proyecto de Intervención "Lectura y Escritura en Educación Básica" (Pilec), desde 1991, hemos realizado trabajo conjunto para la formación de profesores de Educación Básica. 


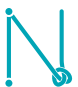

Volumen 5 N. ${ }^{\circ} 42$ enero - junio de 2017 ISSN: 0122-4328 ISSN-E: 2619-6069 pp. $126-137$ pedagógicas y culturales de resistencia vinculadas a la reflexión y transformación de nuestras escuelas y comunidades.

\section{La invitación. Otra perspectiva de formación}

La propuesta de formación la ofrecemos las y los maestros que pertenecemos a la Red de Lenguaje. Los docentes que han participado acuden con la idea convencional de los diplomados y talleres oficiales, en los que no se consideran la experiencia pedagógica y las prácticas del aula. Se han formado con la idea de que los procesos de formación se realizan solo con las lecturas de los textos teóricos y los reportes de las mismas.

Cuando comenzamos el trabajo de formación para las y los maestros, resulta novedoso el planteamiento que les hacemos: "la mayor parte del trabajo se realizará en el aula y las reuniones mensuales del seminario-taller estarán dedicadas a compartir las experiencias, a reflexionar sobre las mismas, a la luz de los textos teóricos y así construir estrategias didácticas para intervenir en el aula". Este planteamiento les agrada. Con esto inicia, para los grupos de profesores, una perspectiva distinta de participación en sus procesos de formación.

\section{La Pedagogía por Proyectos. Compartir las decisiones y la organización del trabajo en el aula junto con los niños}

La Pedagogía por Proyectos es una estrategia de formación en la cual los docentes se van formando en tanto van aprendiendo a construir proyectos de aula junto con los alumnos y padres de familia. Los temas y las actividades de los proyectos son propuestos por los alumnos y se definen en un proceso de negociación y consenso colectivo. A lo largo del proyecto se realizan procesos de reflexión lingüística de los textos que se leen y que se producen en el marco del proyecto; asimismo, se realizan procesos de metacognición de lo aprendido y de evaluación, de manera individual (autoevaluación), colectiva (coevaluación) y sumatoria (realizada por el profesor considerando el proceso del proyecto).

De tal manera que emprender procesos de formación, que los maestros requieren para transformar concepciones de enseñanza y aprendizaje, implica acompañarlos pedagógicamente a fin de construir caminos donde aprendan a convivir con la incertidumbre, transitar hacia la confianza en sí mismos para llevar a cabo prácticas trasformadoras. A continuación, damos a conocer algunos aspectos que vivieron los profesores en este proceso de transformación.

\section{Experiencias y testimonios}

Llevar a cabo los proyectos de aula produjo temor. Plantear a los alumnos que hicieran propuestas sobre los temas para trabajar el proyecto a partir de las preguntas "¿qué vamos a hacer juntos? ¿Qué queremos aprender?", llevó a los maestros a cuestionarse si trabajar asi funcionaria, pues tenían años de experiencia trabajando los contenidos de aprendizaje del programa escolar. Tomaron conciencia de que poseían un discurso contrahegemónico 
y alternativo, pero en realidad continuaban reproduciendo prácticas verticales e impositivas. Se dieron cuenta de que realizar un proyecto requería de tiempo, algunos maestros tardaban tiempo en tomar la decisión, no solo en la forma de enseñar, también en los roles que se asumían en el aula y en los procesos de enseñanza y aprendizaje. Recuperar la experiencia de aula, compartir colectivamente lo que pasaba en el aula, hacía de cada experiencia narrada algo singular. Los siguientes testimonios muestran la problemática que vivieron en la construcción de las prácticas que transforman el trabajo y las relaciones en el aula:

Para mí, en un principio fue un conflicto emocional, laboral, pedagógico, juf!, casi, casi existencial, diría, aceptar aplicarme en el proyecto. ¿Pedagogía por proyectos? ¿Romper esquemas otra vez? Tantas veces en mi carrera profesional han surgido cambios y propuestas que en poco tiempo se consideran obsoletas, ahora nuevamente se presenta otra opción y creo que más descabellada. ¿Ceder el poder de decisión a mis alumnos? No fue fácil aceptarlo. (Profesor Jesús S.).

¡Uy, qué miedo! La verdad, es muy difícil para mi dar el primer paso, no sé cómo iniciar y mucho menos cómo seguir el desarrollo de esto. Tengo pavor, pero sé que si no lo venzo, no podré iniciar algo nuevo con los niños de mi grupo. (Profesora Alicia S.).

A mi me causaba incertidumbre y miedo a que terminara un ciclo escolar y mis alumnos no aprendieran a leer. (Profesora Maria Luisa G.).

Las asesoras acompañantes nos dimos cuenta del miedo que sentían las y los maestros ante lo desconocido, no tenían certeza de cómo reaccionarían los niños ante la pregunta para elegir el tema, cómo asumirían la corresponsabilidad de compartir el poder en el aula, tanto para la organización del trabajo como en la definición de los contenidos a aprender. La resignificación de las relaciones de poder con los niños y la construcción de los conocimientos, a partir del reconocimiento de la pluralidad de conocimientos y saberes, en este caso, saberes de los niños, de los maestros y conocimientos escolares, fueron relaciones que se fueron reestructurando desde una visión de horizontalidad (Santos, 2010, p. 50). Este fue uno de los desafíos que enfrentamos: reestructurarnos como sujetos que construimos y compartimos experiencias pedagógicas desde una visión democrática, horizontal, diversa e inclusiva.

Ahora tengo que abrir los oídos a lo que sabe el niño, ahora tengo que abrir los oídos a lo que sabe el conserje, a lo que sabe el papá, al que sabe el de a lado, dejo de ser el centro del conocimiento para tener que compartirlo con otros y construir un conocimiento colectivo. (Profesora Alma P.).

Recuperar lo que proponían los niños, padres de familia, trabajadores de la escuela fue un aspecto importante en la transformación de las prácticas escolares, implicaba escucharlos y tomar en cuenta lo que decían para integrar la diversidad de saberes en la construcción del proyecto. En este proceso vivimos el diálogo entre los actores, entre los conocimientos y aprendimos a construir consensos. 


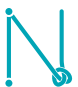

Volumen 5 N. ${ }^{\circ} 42$ enero - junio de 2017 ISSN: 0122-4328 ISSN-E: 2619-6069 pp. $126-137$

\section{Construcción colectiva del proyecto}

Para iniciar la construcción del proyecto, los niños planteaban propuestas sobre temas que deseaban trabajar. Las maestras hacian las preguntas como: ¿Qué vamos a hacer juntos?, y ¿qué vamos a aprender? Al inicio, la elección del tema lo hacian a través de votaciones porque facilitaba el trabajo a las y los profesores, y además era la concepción que se tenía para decidir algo. Con respecto a la práctica de voto mayoritario, algunos niños se quedaban con malestar porque sus propuestas no eran tomadas en cuenta. Tanto maestras como asesoras nos dimos cuenta que estábamos reproduciendo la idea errónea de la "democracia", en la cual solo se toma en cuenta la voz de la mayoría y se silencia a las minorías, así que tuvimos que construir otros caminos para incluir la voz de todos. Uno de ellos fue recurrir a la asamblea, una práctica comunitaria de algunas comunidades oaxaqueñas, de la cual recuperamos la participación colectiva, la puesta en común de las necesidades y la toma de decisiones consensuadas, para construir la unidad respetando los puntos de vista de cada persona.

La experiencia nos fue mostrando que el proceso para la elección del tema era diverso. Había grupos que hacían la elección del tema de acuerdo a una necesidad grupal y en el que no era necesaria la votación, en otros; la elección ocurría de manera consensuada, al integrar las propuestas de los alumnos en un solo proyecto. Asimismo, habia proyectos en los que la elección del tema implicaba un reto por el convencimiento que se tenía que hacer a los compañeros del grupo, para ello los niños argumentaban cada una de sus propuestas.

Una vez elegido el tema, los niños proponían las actividades con la ayuda de las y los maestros. Durante los primeros proyectos sucedieron acontecimientos como los siguientes:

¡Pero qué estoy haciendo! ¡Uf! Qué regadota o metida de pata estaba dando. Había comenzado a realizar actividades que yo proponía y que los alumnos ejecutaban, no estaba tomando en cuenta el interés de los niños. ¿Dónde habia quedado el proyecto de acción? Qué bárbara, me había perdido completamente. (Profesora María Luisa G.).

Durante el proceso las y los maestros reflexionaron sobre la importancia de dar la voz al niño, respetarla, tomarla en cuenta, sobre la necesidad de compartir las decisiones del trabajo en el aula, sobre la importancia de que las y los niños aprendieran a responsabilizarse de su trabajo y aprendizajes. De esta manera, se fueron creando las condiciones para cambiar las relaciones de poder en el aula:

[...] mis alumnos me enseñaron que cuando el niño elige su tema a desarrollar, muestra interés por aprender y por consiguiente se facilita mi trabajo como docente, aún más, si se integra a los padres de familia en las actividades que realizan sus hijos. (Profesora Mercedes L.). 
Una vez que en el grupo se plantearon las actividades, las y los maestros realizaron la planeación didáctica de las mismas. En esta las y los profesores incluyeron los contenidos del programa oficial en el proyecto de los niños, sin forzarlo, en la planificación del proyecto global de aprendizaje. Promovimos que la construcción de currículum se realizara de manera conjunta entre alumnos, maestros y padres de familia. Esta manera horizontal de construir currículum nos ayudó a que todos se sintieran parte de él, y al mismo tiempo, aprendieran a generar lazos de compañerismo y solidaridad.

En el proceso de integración de los contenidos escolares a los proyectos de los niños observamos que en los primeros proyectos las maestras integraban más contenidos del plan de estudios con relación a las actividades propuestas por los niños, pues la preocupación de estas era trabajar los contenidos de las asignaturas marcadas en el bimestre en turno, ya que era una exigencia de los directores, supervisores y, en ocasiones, de los padres de familia.

Las y los profesores se dieron cuenta de que en la planificación de un proyecto no era posible abarcar todas las asignaturas, y que la definición de los contenidos a trabajar en el proyecto se solucionaba dialogando con los niños para llegar a acuerdos sobre lo que se iba a aprender. Los contenidos del programa que no se podían trabajar a través de los proyectos se abordaban al margen de los proyectos.

Mi objetivo era terminar los contenidos que marca el programa" en muchas ocasiones los contenidos eran vistos de pasada sin llegar a profundizar los temas, pero sobre todo yo era la que daba la clase, sin detenerme a pensar si era significativa la enseñanza y el aprendizaje que recibian mis alumnos, o tal vez intentaba darme cuenta, pero como ya era mi costumbre, seguía con mi forma de trabajo.

[...] me fui dando cuenta de muchos errores incurridos al ir leyendo, comentando y analizando las experiencias que narran las maestras sobre la Pedagogía por Proyectos y los cambios significativos para los alumnos. Me di cuenta que esta nueva forma de trabajo es más humanista y digna porque lo más valioso, es tomar en cuenta a los niños y niñas sobre cómo y qué quieren que se trabaje con ellos. (Profesora Silvia L.)

Durante el desarrollo del proyecto se realizaron actividades propuestas por los niños y muchas de estas se compartieron con estudiantes de otros grados, padres de familia y personas de la comunidad. Las y los maestros se dieron cuenta de los cambios en sus prácticas de enseñanza y del cambio de actitud en los niños, su disposición y entusiasmo para aprender y realizar las actividades del proyecto fue un cambio muy significativo.

Con la Pedagogía por Proyectos los niños saben, proponen, les gusta que los tomemos en cuenta, eso es algo que me ha motivado a hacer cambios en mi práctica... (Profesora Mercedes L.).

Trabajar con la Pedagogía por Proyectos es una buena alternativa ya que parte del interés y de la curiosidad del estudiante, los contenidos se apegan a su realidad, esto hace que los aprendizajes sean significativos. Trabajan en equipo, reflexionan, 


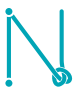

Volumen 5 N. ${ }^{\circ} 42$ enero - junio de 2017 ISSN: 0122-4328 ISSN-E: 2619-6069 pp. $126-137$ construyen su conocimiento, buscan información y respuestas a sus interrogantes, se involucran en el diseño de las actividades y se crea un ambiente propicio para el aprendizaje. (Profesora Elia R.).

Tanto en la Pedagogía por Proyectos como en la Red de Lenguaje planteamos el principio de horizontalidad, entendido como el valor de igualdad que todos tenemos en el grupo. El poder transita de uno a otro lado y no se centra en una persona. En las reuniones aportamos desde nuestras subjetividades, compartimos desde lo teórico y desde la experiencia. Trabajamos para no sentirnos menos que el otro o los otros.

Para reflexionar sobre el principio de horizontalidad retomamos el concepto de reciprocidad que Jaime Martínez Luna plantea para dar a conocer la manera en que las comunidades realizan el intercambio, tanto de saberes como de trabajo. La reciprocidad se nombra de distintas maneras en cada pueblo, es el acto de dar y recibir sin sentirse más que el otro. En algún momento se puede tener la satisfacción de dar o el beneficio de recibir.

En nuestro proceso de formación promovimos que la reciprocidad se viviera día a día a través de compartir experiencias, ideas, acompañamiento en las aulas. Reflexionamos que no se trataba de sentirnos el centro del conocimiento en nuestro trabajo con los niños y padres de familia, sino aprender a compartir los cargos de poder, con la conciencia de que algunas veces podíamos ayudar y otras auxiliarnos con la experiencia de los demás. Reflexionamos que en nuestras relaciones horizontales todavía persisten las relaciones de poder y que uno de nuestros aprendizajes sería transitar hacia relaciones de reciprocidad.

\section{Las relaciones entre niños, padres de familia y maestros se transforman}

La práctica de la Pedagogía por Proyectos generó cambios en las relaciones de poder entre maestros y alumnos al construirse un ambiente que priorizó el trabajo cooperativo, la solidaridad y la democracia en el aula.

Veo cambios en los niños de primero que no querian hablar, ahora ellos se reúnen, unos se abrazan, otros se sientan, de repente hay un montón de niños y niñas ahí discutiendo lo que van a decir para convencer a los demás, esa parte ya la hacen independientemente. (Profesor Cristiam S.).

Por otra parte, los niños construyeron la confianza para decirnos lo que querían hacer, sin el temor a ser regañados o a minimizar sus opiniones. En ocasiones, la presión ejercida por los alumnos para trabajar Pedagogía por Proyectos nos orillaba a transformar nuestras prácticas de enseñanza para no regresar a las prácticas tradicionales:

Veníamos de leer el cuento "La lechera" y se me ocurrió hacer un alto y trabajar, con toda normalidad y en el ejercicio del poder, el proyecto de lectura "Crisantemo". Conseguí interesar a los niños, participaron activamente, desarrollé algunos contenidos, después de varias sesiones y casi al término del proyecto, Levi se acercó a mi escritorio para decirme: 
- ¿Por qué primero nos hablas de una cosa y luego de otra? -.

-¿Por qué? -, pregunté intrigado.

-Porque primero estábamos con el proyecto de "La lechera" y nos faltaban algunas cosas, y luego nos leíste "Crisantemo" y hablamos de verbos y todo eso. Dijiste: hagan esto, hagan el otro, pero no has preguntado qué queremos hacer y además nos falta el proyecto de cocinar ¿Cuándo lo vamos hacer?

Por un momento, mi mayor deseo fue que nadie más hubiera escuchado la conversación, pero cuando volteé me di cuenta que otros niños me apuntaban con su mirada acusadora. Las orejas me ardieron un poco, sobre todo porque entre ellos estaba Edwin y sabía que si él había escuchado, seguro se armaría un revuelo. Lo único que atine a decir fue:

-Bueno, es que eso les toca a ustedes, esa es su responsabilidad, si no exigen, pues no pasa nada y no hacemos nada, como en todo. No pude detener a Edwin:

-Pues hagamos asamblea y que digan los demás-, dijo. Los demás niños lo apoyaron. (Profesor Cristiam S.).

Nuestro principal reto fue sostener nuestras prácticas innovadoras, y aunque lo intentamos, siempre existían oportunidades para abandonarlas. De pronto ya no trabajábamos el proyecto de los niños y regresábamos a lo habitual, cubrir los contenidos del programa escolar y retornar a la enseñanza vertical y tradicional. Estas experiencias nos ayudaron a reflexionar sobre nuestras prácticas, en lo individual, con nuestros pares y asesoras. Nos dimos cuenta que el acompañamiento nos daba fuerza y voluntad para continuar con el proceso de cambio y no sentirnos solos en el camino de la transformación pedagógica.

Otro cambio que se manifestó entre las y los profesores fue la relación con la comunidad. En algunos casos logramos establecer un diálogo de saberes, entre saberes propios que emergen de la comunidad, como parte de su cultura, y los conocimientos escolares. En la siguiente experiencia los alumnos decidieron recuperar los conocimientos propios e integrar a personas de la comunidad.

El proyecto era conocer el proceso de elaboración de la tortilla, pero ellos quisieron saber sobre los tipos de maíz que había en la comunidad y cómo se sembraban. Eran conocimientos que ya sabian las mamás. Vi cómo las señoras les explicaban a los niños los tipos de maíz y cómo se hacía para poner el nixtamal. Todos estos procesos los fui aprendiendo junto con ellos hasta el día en que toda la comunidad se reunió para dar a conocer el proceso de elaboración de la tortilla. Los niños molieron el nixtamal en molino de mano porque la comunidad es muy pequeña y no hay molino, pero fue tan bonito porque llegaron las abuelitas a convivir. Se hicieron enfrijoladas y los niños se hicieron cargo de esto. (Profesora Marisol S.).

En el trabajo en colectivo ya no somos la única fuente de información, compartimos este papel con los niños, con los padres, con los abuelos, con las personas de la comunidad. Intentamos no hegemonizar el conocimiento ni el 


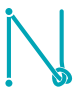

Volumen 5 N. ${ }^{\circ} 42$ enero - junio de 2017 ISSN: 0122-4328 ISSN-E: 2619-6069 pp. $126-137$ poder en el aula, y vamos perfilando nuestro trabajo hacia la construcción de una comunidad donde se practica la reciprocidad como forma de vida.

Las experiencias que vivimos en cada aula se compartieron en las sesiones del seminario, esto nos ayudó a apropiarnos de esta forma de trabajo, a reflexionar y cuestionar las prácticas que no resultaron pertinentes para enseñar. Avanzamos en la transformación de nuestras prácticas pedagógicas por la disposición para emprender un proceso de cambio y por el acompañamiento en colectivo que construimos maestros y asesoras. Para todos significó construirnos a si mismos como maestros.

\section{Las resistencias}

Durante el desarrollo del diplomado vivimos resistencias, una de ellas fue al cambio a abandonar las prácticas de enseñanza tradicionales y a dejar de trabajar con el programa escolar como única posibilidad de enseñanza y aprendizaje. Tomamos conciencia de que la comodidad da seguridad porque no requerimos de aventurarnos hacia lo desconocido. Aunado a esto, reflexionamos que los cursos de actualización que implementa la Secretaría de Educación Pública no son pertinentes a las necesidades de nuestra formación y al paso del tiempo los cursos de actualización y capacitación quedan obsoletos. Por tal razón, los cursos no son atractivos y no cubren nuestras expectativas. La Pedagogía por Proyectos nos hizo enfrentarnos a situaciones poco comunes que se salian de nuestro control habitual, situación que no todos estuvimos dispuestos a vivir por las estructuras rígidas en las que fuimos educados y que seguimos reproduciendo.

Al enterarme de la propuesta para iniciar Pedagogía por Proyectos, pensé en uno más de tantos cursos que se han generado sobre los escritorios de supuestos pedagogos, que movidos por la justificación de su salario, inventan programas burocráticos. (Profesor Jesús S.).

La práctica cotidiana me había ubicado en una zona de confort de la cual no deseaba salir así tan fácilmente. ¿Por qué?, por los múltiples "beneficios" que me provocaba realizar un trabajo cotidiano que se me había vuelto rutinario, una serie de pasos los cuales podía llevar a cabo año tras año sin hacer el más mínimo esfuerzo por cambiar. (Profesor Rodolfo C.).

El rechazo de los padres de familia a las propuestas pedagógicas alternativas fue otra resistencia que identificamos. Es común en las comunidades que los padres de familia estén al pendiente de lo que sucede en las aulas. Esta situación genera inseguridad porque no sabemos qué reacciones tendrán frente a la situación de que sus hijos trabajarán con una propuesta pedagógica diferente a la tradicional. Sabíamos que los padres de familia vienen de historias escolares con prácticas tradicionales. Nos enfrentamos con esas historias, ellos nos comentaban que trabajar con proyectos no funcionaba, que ellos preferían que sus hijos aprendieran con las prácticas de enseñanza conocidas por ellos. Estas situaciones nos llevaron a plantearnos la necesidad de emprender procesos de formación con los padres de familia para integrarlos en la construcción y participación en los proyectos de los niños. 
Cómo va a haber respeto si se les pregunta libremente a los niños qué es lo que quieren hacer. La escuela no está para preguntar sino para enseñarle a nuestros hijos a leer y escribir, a hacer las cuentas, si no para qué vienen a la escuela. (Padre de familia de la Sierra Norte de Oaxaca).

Me parece que es mejor que sigan los libros tal como vienen o comprarles una guía. Veo que mi hijo aprende más asi y no con los proyectos que se llevan más tiempo. Si aprenden, pero es más trabajo para nosotros. (Padre de familia de Valles centrales de Oaxaca).

Otra forma de resistencia que logramos identificar, con una connotación diferente, fue la resistencia pedagógica, asumida frente a los embates de la política educativa y sus programas escolares homogeneizantes. La necesidad de construir alternativas pedagógicas contextualizadas que toman en cuenta a los alumnos, a los padres de familia, a la comunidad; que valoran los saberes comunitarios, las prácticas comunales, las decisiones colectivas, las asumimos como formas de resistencia para construir una escuela a la altura de las necesidades pedagógicas, culturales y sociales de las comunidades.

\section{Reflexiones}

Los procesos que vivimos durante el trabajo con la Pedagogía por Proyectos nos permitieron transformarnos, aprender a enfrentar situaciones que obstaculizaban el desarrollo de los proyectos de aula y de nuestra formación profesional. Aprendimos que las escuelas se van construyendo con sus propios procesos, con la participación de los maestros, niños y padres de familia, a pesar de las demandas que plantea la institución escolar; que los cambios posibles en las escuelas y aulas, depende de la disposición que tengamos para emprender procesos de formación colectivos con propósitos de transformación, con perspectiva crítica frente a las políticas educativas coercitivas.

\section{Referencias}

Aboites, H. (2012). La medida de una nación. Los primeros años de la evaluación

en México. Historia de poder y resistencia (1982-2012). Ciudad de México: Clacso, UAm-X, UAM-I, Editorial Íthaca.

Hernández, L. (2013) No habrá recreo. Contra-reforma constitucional y desobediencia magisterial. México D.F.: Rosa Luxemburg Stiftung y Para Leer en Libertad Ac.

Palermo, Z. (2014). Para una Pedagogía Decolonial. Buenos Aires: Del signo.

Ruíz, C., Pulido, R., Ruíz, P., González, T., Chona, J., Ortíz, G., y Medoza, M. (agosto de 2010). Diplomado: La enseñanza de la lengua desde la Pedagogía por Proyectos en educación básica. Distrito Federal, México.

Santos, de S., B. (2010). Descolonizar el saber, reinventar el poder. Montevideo: Trilce/ Extensión universitaria. 